\title{
An Msh3 ATPase domain mutation has no effect on MMR function
}

\author{
Yasmin Edwards* (1)
}

\begin{abstract}
Objective: To demonstrate that the Msh3 ATPase domain is required for DNA mismatch repair and tumor suppression in a murine model.

Results: The DNA mismatch repair proteins are members of the ABC family of ATPases. ATP binding and hydrolysis regulates their mismatch repair function. In the current study, a mouse model was generated harboring a glycine to aspartic acid residue change in the Walker A motif of the ATPase domain of Msh3. Impaired ATP mediated release of the Msh2-Msh ${ }^{G D / G D}$ complex from it's DNA substrate in vitro confirmed the presence of an ATPase defect. However, the mismatch repair function of the protein was not significantly affected. Therefore, mutation of a critical residue within the ATPase domain of Msh3 did not preclude mismatch repair at the genomic sequences tested. Indicating that Msh3 mediated mismatch function is retained the absence of a functional ATPase domain.
\end{abstract}

\section{Introduction}

DNA mismatch repair (MMR) proteins target and mediate repair of DNA polymerase errors of replication and signal the DNA damage response [1]. The MMR system consists of the highly conserved MutS and MutL homologues. In eukaryotes, the MutS homologues are Msh2, Msh3 and Msh6, which function as heterodimers. The MutS $\alpha$ heterodimer (Msh2-Msh6) targets single base mispairs and single base insertion/deletions for repair, and the MutS $\beta$ heterodimer (Msh2-Msh3), overlaps in the repair of single base insertion/deletions, but primarily targets larger insertion/deletions for repair $[2,3]$. MutS heterodimers recruit the MutL homologues (Mlh1, Pms2, Mlh3) to mediate the next steps of repair [4]. Mutations in MSH2, MSH6, MLH1 and PMS2 have been identified in Hereditary non-polyposis colorectal cancer/ Lynch Syndrome (HNPCC)/LS), a familial cancer syndrome [4]. Mutations in the $M S H 3$ gene have not been associated with Lynch Syndrome.

The ATPase domain is highly conserved and regulates the affinity of the MutS proteins for their DNA substrates [5]. Msh2 and Msh6 murine models defective in ATPase function have been generated $[6,7]$. These mutations prevented

*Correspondence: Yasmin.edwards@bcc.cuny.edu

Bronx Community College, 2155 University Avenue, Bronx, NY 10453, USA
ATP mediated release of the DNA substrate leading to, reduced DNA MMR, increased tumorigenesis and reduced lifespans. The impact of an Msh3 ATPase defect has not been determined in a murine model. The aim of this study was to analyze the $M s h 3^{G 855 D}$ mouse line, which harbors a glycine to aspartic acid mutation in the ATPase domain of the protein, on MMR, tumor suppression and survival.

\section{Main text \\ Methods \\ Animals}

A mutation was introduced into exon 20 of the $M s h 3$ gene changing codon 855 from glycine (GTT) to aspartic acid (GAC) using site directed mutagenesis (Stategene, Quick change kit), following sub-cloning from a bacterial artificial chromosome. The mutation was confirmed by sequencing. A NotI fragment containing loxP sites on either side of a neomycin-PGK hygromycin resistance cassette was subcloned into an EcoRI site 150 bp upstream of exon 20. The EcoRI fragment was re-subcloned into the $(+)$ pBluescript vector containing Msh3 genomic DNA. The vector was linearized and used to modify the Msh3 genomic locus via gene targeting in G4 embryonic stem cells (ES). Positive ES clones were identified via PCR and the correct integration confirmed using long range PCR and southern blot analysis. Positive ES cell clones were injected into C57BL6/6J 
females (Jackson Laboratories). One transmitted the mutant allele through its germ line. F1 males carrying the mutant allele were mated to $\mathrm{Zp} 3$ Cre transgenic females (C57BL/6J purchased from Jackson Laboratories), resulting in deletion of the resistance cassette by loxp mediated recombination. F1 Heterozygotes carrying the modified allele were intercrossed producing $\mathrm{n}=20, \mathrm{Msh}^{+/+}, 30$, $M s h 3^{G D /+}$ and $\mathrm{n}=22, M s h 3^{G D / G D}$ mice. Cohen's effect size value of $(\mathrm{d}=0.7) . M s h 3^{-/-}$animals utilized as negative controls were gifts from the laboratory of Dr. Winfried Edelmann [8]. All animals were maintained at the Albert Einstein College of Medicine animal care facility in accordance with Institutional Animal Care and Use Committee (IACUC) guidelines.

\section{Electromobility shift assay (EMSA)}

The effect of the $M s h 3^{G D / G D}$ mutation on ATPase mediated DNA substrate release, was examined in nuclear extracts from each genotype. Nuclear extracts were prepared as described [9]. Nuclear extracts were prepared from pooled mouse testes obtained from five animals of each genotype $M s h 2-M s h 3^{+/+}, M s h 3-M s h 3^{G D /+}$, Msh2$M s h 3^{G D / G D}$ animals. Testes were minced, washed twice in cold PBS and centrifuged in $15 \mathrm{ml}$ conical tubes for $4 \mathrm{~min}$ at $4000 \mathrm{rpm}$. Pellets were re-suspended in $5 \mathrm{vol}-$ umes of low salt buffer (10 mM HEPES, $1.5 \mathrm{mM} \mathrm{MgCl}$, $10 \mathrm{mM} \mathrm{KCl}, 0.5 \mathrm{mM}$ DTT and $0.5 \mathrm{mM}$ PMSF), incubated on ice for $10 \mathrm{~min}$, and centrifuged at $4{ }^{\circ} \mathrm{C}$ for $10 \mathrm{~min}$ at $4000 \mathrm{rpm}$. Pellets were re-suspended in 2 volumes of the low salt buffer, homogenized in a dounce 10-20 times and centrifuged at $4{ }^{\circ} \mathrm{C}$ for $20 \mathrm{~min}$ at $14,500 \mathrm{rpm}$ in a Sorval. The pellet was re-suspended in high salt buffer $(20 \mathrm{mM}$ HEPES, 25\% (v/v) glycerol, $420 \mathrm{mM} \mathrm{NaCl}, 1.5 \mathrm{mM} \mathrm{MgCl}_{2}$ ) 2 mM EDTA, 0.5 DTT, 0.5 mM PMSF,) and homogenized 3-4 times. Following homogenization, samples were incubated for $30 \mathrm{~min}$ at $4{ }^{\circ} \mathrm{C}$ while stirring. The mixture was centrifuged at $14,500 \mathrm{rpm}$ in a Sorval at $4{ }^{\circ} \mathrm{C}$ for $20 \mathrm{~min}$. The supernatant was dialyzed overnight at $4 \mathrm{C}$ in dialysis buffer (20 mM HEPES, 20\% (v/v) glycerol, $100 \mathrm{mM}$ KCL, 0.2 mM EDTA, 0.5 mM DTT, 0.5 mM PMSF). The supernatant was centrifuged at $14,500 \mathrm{rpm}$ in a Sorval for $20 \mathrm{~min}$ at $4{ }^{\circ} \mathrm{C}$. The nuclear extracts were aliquoted and stored at $-80^{\circ} \mathrm{C}$. To prepare binding reactions: the sense strand oligonucleotide $5^{\prime}$ GCTTAGGATCATCGAGGATCGAGCTCGGTGCAATTCAGCGG- CA, and antisense oligonucleotide insert $5^{\prime}$ CCGCTGAATTGCACCGAGCTCCACACACAGAT TCCTCGATGATCCTAAGC $3^{\prime}$ and homoduplex oligonucleotide $5^{\prime}$ CCGCTGAATTGCACCGAGCTCGATCCTCGATGATCCTAAGC were end labeled with infrared-700 (IR700) and annealed in $1 \times$ T4 Kinase buffer with $3 \times$ molar ratio of antisense oligonucleotide containing a $4 \mathrm{CA}$ insert. Thirty micrograms of nuclear extract was pre-incubated in $1 \times$ DNA binding buffer, 1 ug of poly $(\mathrm{dI}-\mathrm{dC})$ and $1 \mathrm{ng}$ of unlabeled homoduplex for $5 \mathrm{~min}$ on ice in a total volume of 19ul. Five nanograms of radio labeled DNA probe was added and the mixture incubated at room temperature in the dark for $20 \mathrm{~min}$. For cold competitor reactions, the cold competitor was added in the preincubation reaction. ATP mediated release was induced by adding ATP 15 min following addition of DNA. The binding reaction mixture was electrophoresed on a $5 \%$ polyacrylamide gel in $0.5 \times$ trisborate EDTA (TBE) buffer. The gel was imaged using the Odyssey Infrared Imaging System (LI-COR). Image J software was use to analyze the binding intensities graphed in the binding curves.

\section{Microsatellite instability analysis}

Microsatellite sequences were analyzed by single cell PCR to determine the effect of Msh3 $3^{\text {G855D }}$ mutation on microsatellite stability. Equal amounts of tail DNA from five mice of each genotype $\left(M s h 3^{+/+}, M s h 3^{G D / G D}\right.$, and $M s h 3^{-1-}$ ) were pooled separately and diluted to between 0.5 and 1.0 genome equivalents. DNA was extracted from tumors and amplified for MSI analysis (not all tumors amplified). PCR cycling parameters for U12235, D17MIT91 and (TG) 27 markers were performed as previously described [10]. A $95{ }^{\circ} \mathrm{C}$ for $1 \mathrm{~min}, 57-63^{\circ} \mathrm{C}$ for $1 \mathrm{~min}$, and $72{ }^{\circ} \mathrm{C}$ for $1 \mathrm{~min}$ for 30 cycles and $72^{\circ} \mathrm{C}$ for 5 min once. The products were diluted by loading buffer, heated at $95{ }^{\circ} \mathrm{C}$ for $5 \mathrm{~min}$, and loaded onto $6 \%$ vertical polyacrylamide gels. Following electrophoresis, gels were fixed, dried, and exposed to X-ray film overnight $(12 \mathrm{~h})$ to 2 days. A single observer analyzed samples and a second observer reviewed equivocal samples.

\section{Histopathological analysis of tumors}

Moribund mice were identified and sacrificed using $\mathrm{CO}_{2}$ according to IACUC protocols. Tumors were removed and fixed in $10 \%$ neutral buffered formalin. Tumors were then embedded in paraffin and sections were removed for hematoxylin and eosin staining and analyzed.

\section{Statistical analysis}

The Kaplan-Meier survival curve was generated and analyzed using Graphpad prism 3.0 software. Microsoft Excel version 14.7.6 was used to determine the $\mathrm{p}$ values between $M s h 2-M s h 3^{G D / G D} M s h 32-M s h 3^{+/+}$and $M s h 2-M s h 3^{-/-}$somatic MSI using the student $\mathrm{t}$ test. The Fisher exact test was used to determine the significance of tumor MSI rates. G*Power software version 3.1 was used to analyze sample size significance. Differences were determined to be statistical significant at $p$ values $<0.05$. 


\section{Results}

Msh2-Msh3 $3^{G D / G D}$ impaired ATP mediated DNA substrate release

Dissociation of the Msh2-Msh3 ${ }^{+/+}$(positive control) and $M s h 2-M s h 3^{G D / G D}$ complexes from the DNA substrate was observed upon addition of increasing concentrations of cold competitor to the binding reactions, confirming the specificity of binding (Fig. 1a). The addition of increasing concentrations of ATP to the binding reactions, induced release of the Msh2$M s h 3^{+/+}$heterodimer from the substrate, while the $M s h 2-M s h 3^{G D / G D}$ heterodimer persisted at the highest ATP concentrations (Fig. 1b). At physiological ATP concentrations of approximately 3-5 $\mathrm{mM}$ [11], the mutation reduced ATP induced DNA substrate release. Preliminary titration reactions were completed as shown in Additional file 1.
Somatic microsatellite instability in Msh2-Msh $3^{G D / G D}$ animals Microsatellite instability is a hallmark of MMR deficiency [12]. Somatic MSI in the Msh2-Msh $3^{G D / G D}$ animals was compared to Msh2-Msh ${ }^{+/+}$and Msh2-Msh3 $-/-$ animals (the positive and negative controls). At the $\mathrm{TG}_{27}$ and D7Mit91 dinucleotide markers, the highest instability was observed in the $M s h 2-M s h 3^{-1-}$ animals, 15 and $9 \%$ respectively, with $\mathrm{p}$ values of 0.03 and 0.02 compared to wild type (Table 1 ). Conversely, no significant instability was observed in the Msh2-Msh $3^{G D /}$ $G D$ and $M \operatorname{sh} 2-M \operatorname{sh} 3^{+/+}$animals, at the dinucleotide markers. Indicating that the $M s h 2-M s h 3^{G D / G D}$ complex retained MMR function at the dinucleotide repeat

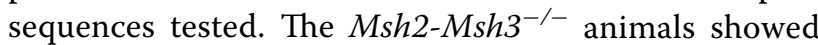
no instability at the mononucleotide marker (Table 1 ). While MSI in the Msh2-Msh $3^{G D / G D}$ animals was significantly increased compared to $M s h 2-M s h 3^{+/+}$animals
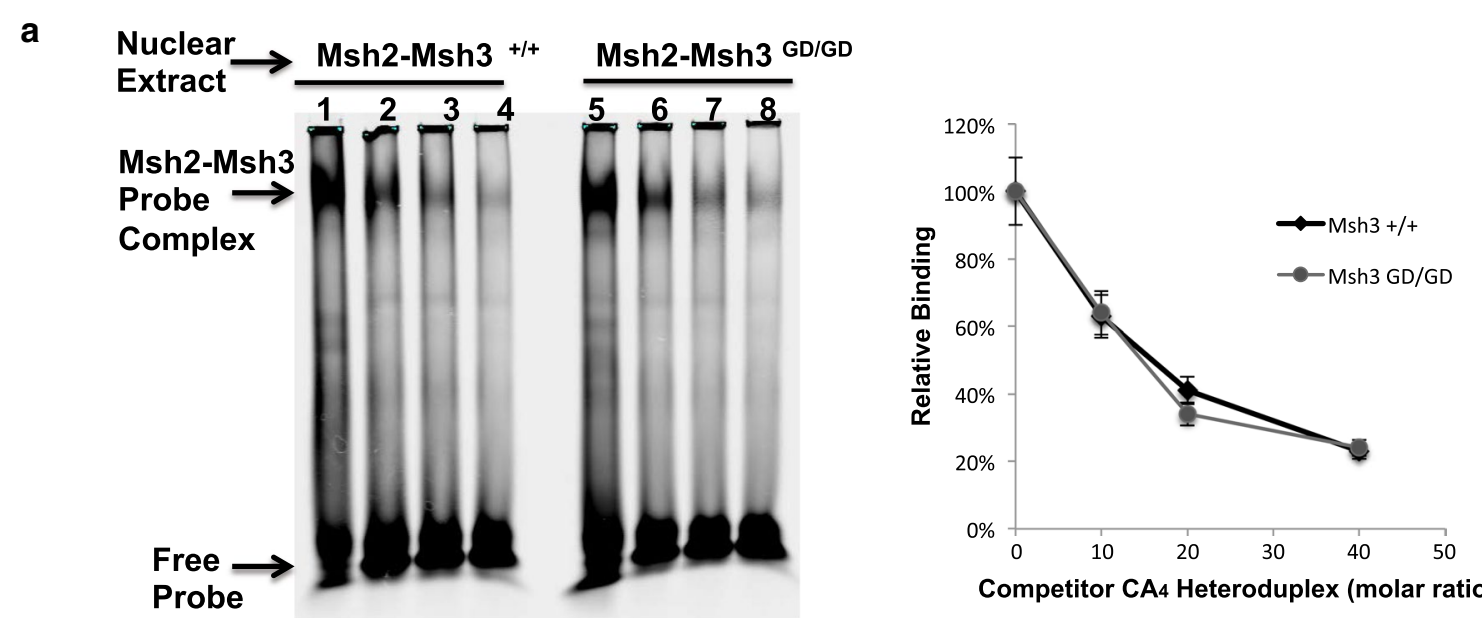

Competitor CA4 Heteroduplex (molar ratio)
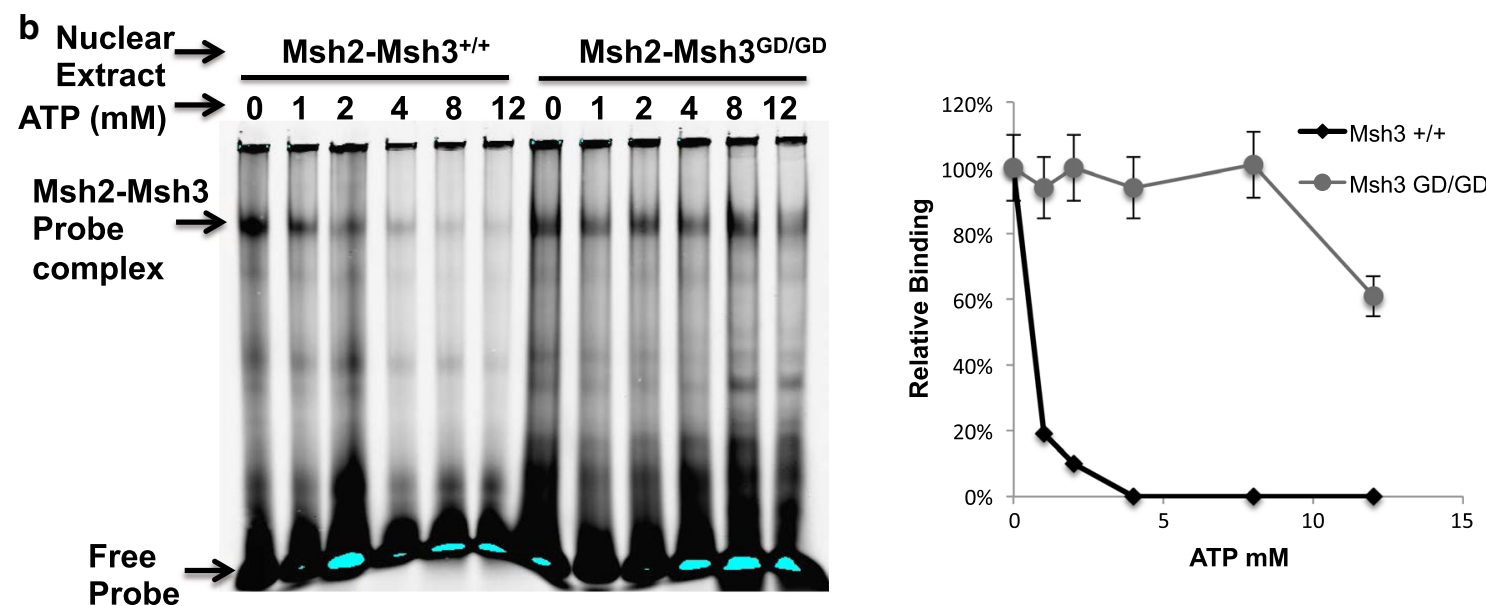

Fig. 1 Electromobility shift assays. a Increasing concentrations of cold CA 4 competitor induced substrate release by both Msh2-Msh3 ${ }^{+/+}$and Msh2-Msh3 $3^{G D / G D}$ heterodimers. Bars indicate that there is less than 10\% difference between Msh2-Msh3 ${ }^{\mathrm{GD} / \mathrm{GD}}$ binding compared to wild type except at 20X molar ratio. b Increasing ATP concentration resulted in release of DNA binding in the Msh2-Msh3 ${ }^{+/+}$but not the Msh2-Msh3 ${ }^{\text {GD/GD }}$ nuclear extracts. Percent difference bars (set at 10\%) show no overlap in binding upon addition of ATP 
Table 1 Somatic and tumor MSI

\begin{tabular}{|c|c|c|c|}
\hline Marker & Msh2-Msh3 $3^{+/+}$ & Msh2-Msh3 $3^{\text {GD/GD }}$ & Msh2-Msh3 ${ }^{-1-}$ \\
\hline \multicolumn{4}{|l|}{ Somatic MSI } \\
\hline U12335(A) & $(5 / 95) 5 \%$ & (16/72) 22\% & (1/99) $1 \%$ \\
\hline $\mathrm{TG}_{27}$ & $(3 / 90) 3 \%$ & $(8 / 95) 8 \%$ & (11/75) $15 \%$ \\
\hline D7Mit91 & (0/77) $0 \%$ & $(1 / 77) 1 \%$ & (7/79) 9\% \\
\hline \multicolumn{4}{|l|}{ Tumor MSI } \\
\hline U12335 & $(0 / 6) 0 \%$ & (4/8) 50\% & $(2 / 15) 13 \%$ \\
\hline $\mathrm{TG}_{27}$ & $(0 / 6) 0 \%$ & $(1 / 8) 13 \%$ & (4/8) 50\% \\
\hline
\end{tabular}

Somatic and tumor microsatellite instability: MSI was analyzed using the mononucleotide marker U12235n and dinucleotide markers $\mathrm{TG}_{27}$ and D7Mit91

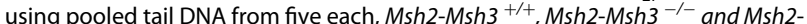
Msh3 ${ }^{\text {GD/GD }}$ mice, (p values U12335: $\mathrm{Msh}^{+/+}$vs. Msh3 ${ }^{\mathrm{GD} / \mathrm{GD}}=0.036$ and $\mathrm{Msh}^{+/+}$: $\mathrm{Msh}^{-1-}=0.02$, $\mathrm{p}$ values $\mathrm{TG}_{27}: \mathrm{Msh}^{+/+}$vs. Msh3 ${ }^{\mathrm{GD} / \mathrm{GD}}=0.06$ and $\mathrm{Msh}^{+/+} \mathrm{vs}$. $\mathrm{Msh}^{-1-}=0.038, \mathrm{p}$ values D7Mit91: $\mathrm{Msh}^{+/+}$vs. Msh3 $\left.{ }^{-1-}=0.05\right)$

at the U12235 mononucleotide marker ( $\mathrm{p}$ value 0.03 ). At the dinucleotide sequences, the $M s h 2-M s h 3^{G D / G D}$ animals were similar to wild type and not the Msh2$M s h 3^{-/-}$animals. Confirming that Msh3 MMR function remained. Tumor MSI was tested at the U12235 (A) $\mathrm{n}$ and $\mathrm{TG}_{27}$ markers (no instability was observed at the D7Mit91 marker in the tumors tested). Comparison of MSI negative tumors from wild type animals to the tumor numbers in the $M s h 2-M s h 3^{G D / G D}$ animals proved significant using the Fisher exact test, $\mathrm{p}$ value $=0.03$. MSI in tumors was similar to the somatic MSI, with greater instability at the mononucleotide marker in the $M s h 2-M \operatorname{sh} 3^{G D / G D}$ tumors and at the dinucleotide marker in the Msh2-Msh3 ${ }^{-/-}$tumors. These numbers were not significant (Table 1).

\section{Late stage microsatellite unstable tumors observed with no significant loss of survival}

Analysis of tumors revealed GI tumors, lymphomas and other tumors Fig. 2a. Low tumor numbers were consistent with a weak tumor phenotype in the $M s h 2-M s h 3^{G D / G D}$ animals revealing no significant difference compared to Msh2-Msh3 ${ }^{+/+}$tumorigenesis. The Msh2-Msh3 $3^{G D / G D}$ tumors showed both contractions and expansions (Fig. 2b). There was no significant reduction in survival between $M s h 2-M s h 3^{G D / G D}$ animals (21 months) compared to wild type animals (24 months), Fig. 2c. Msh2-Msh $3^{G D / G D}$ animals succumbed to tumors starting at 12th months, similar to the tumor onset previously reported in Msh2-Msh3 ${ }^{-/-}$mice [8]. Wild type animals began to succumb later, at 16th months (Fig. 2c).

\section{Discussion}

ADP binding in the nucleotide pocket of the Msh3 ATPase domain, is thought to increase the affinity of the Msh2-Msh3 heterodimer for DNA, while ATP binding and hydrolysis initiates recruitment of the MutL proteins and additional repair factors required to complete repair and ultimately release the substrate [13-15]. Perversely, in the current study a defective Msh3 ATPase domain did not abrogate the MMR function of the Msh2-Msh3 complex. One study, suggested that the MSH3 subunit of the heterodimer was most involved in targeting and binding of the insertion substrate, while ATP binding in the MSH2 subunit initiated the recruitment of the MutL proteins to begin the final steps of repair and release [13]. As this model would predict, the $M s h 2-M s h 3^{G D / G D}$ complex bound to the DNA substrate, confirming that DNA binding was not impaired by the mutation. While a defect in ATP induced release of the $\mathrm{CA}_{4}$ substrate was confirmed. The EMSA binding reactions were conducted in vitro and the timeline of the binding stoichiometry was not explored. Repair at dinucleotide sequences confirmed Msh3 MMR function, notwithstanding the inability to hydrolyze ATP in the Msh $3^{\mathrm{GD} / \mathrm{GD}}$ subunit. A functional Msh2 ATPase domain appeared sufficient to promote delayed release from the DNA substrate, facilitating the final steps of repair in vivo.

MMR function was not significantly impaired in the $M s h 3^{G 855 D}$ mouse line. The mutation led to increased instability at the genomic mononucleotide repeat sequence, and in the tumors tested. Some Msh2-Msh3 ${ }^{G D /}$ $G D$ animals succumbed to these tumors at earlier ages than did wild type animals, but the survival rates were not significantly reduced. The results suggest that Msh3 is not a primary driver of tumorigenesis. The Msh2$M s h 3^{G D / G D}$ phenotype shows greater similarity to late onset sporadic tumorigenesis and not the familial Lynch syndrome with which mutations in the MSH2 and MSH6 MutS homologues are associated [16].

\section{Limitations}

- ATP was added in the final 5 min of the binding reactions and so the effect of lengthier binding reaction times on DNA substrate release dynamics in the presence of ATP was not evaluated.

- The inclusion of additional dinucleotide markers, in addition to trinucleotide and tetranucleotide genomic repeat markers would be more informative in light of Msh3's role in maintaining stability at these sequences. 
a
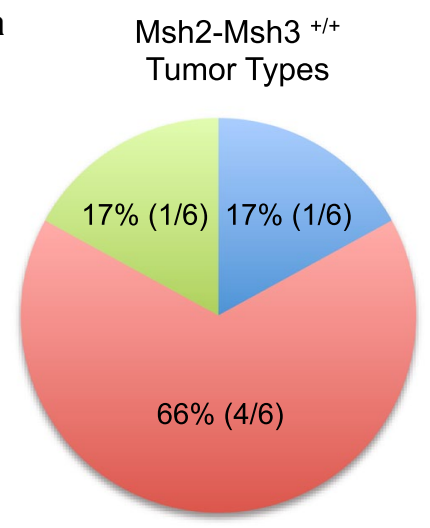

b

Examples of Tumor MSI

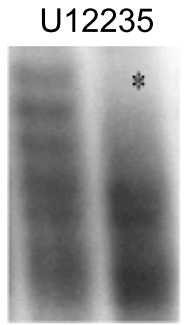

Normal Tumor

\section{D7Mit91}

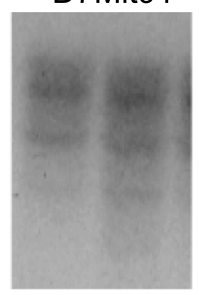

Normal Tumor

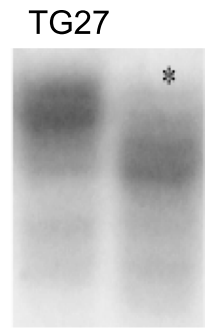

Normal Tumor
- Gl Tumors

- Lymphomas

Other

Fig. 2 Tumor phenotype and survival curve. a Tumor histopathology was determined in Msh2-Msh3 ${ }^{+/+}$and Msh2-Msh3 $3^{G D / G D}$ animals. Gastrointestinal tumors in the Msh2-Msh3 ${ }^{+/+}$animals included an adenoma and the other tumors included a histiocytic sarcoma. The Gl tumors in the Msh2$M s h 3^{G D / G D}$ animals included an adenoma, carcinoma and a polypoid adenomadous hyperplasia, the other tumors included two hepatic carcinomas, an extramedullary myeloma, bronchio-alveolar adenoma and two hepatic adenomas. b Tumor microsatellite amplifications show contractions in Msh2-Msh3 ${ }^{\mathrm{GD} / \mathrm{GD}}$ tumors, no MSI was observed using the D7Mit91 marker in the tumors tested. c Survival curve represents the time of death or the time at which the mice became moribund and were sacrificed. The survival curves were generated using Graphpad prism 3.0 software. The black line, Msh2-Msh3 ${ }^{+/+}$mice $(n=20)$, the grey line represents the Msh2-Msh3 ${ }^{G D / G D}(n=22)$ animals. The 50\% survival was found to be 21 months in the Msh2-Msh3 ${ }^{\mathrm{GD} / \mathrm{GD}}$ animals, $\mathrm{p}$ value $>0.05$

\section{Additional file}

Additional file 1. Preliminary electromobility shift assay (EMSA)—Preliminary EMSA including negative control (lane 1) and fixed concentrations of cold competitor (30x) in lanes 5, 6 and 7. ATP (4 mM) with Msh2-Msh3 ${ }^{+/+}$, Msh2-Msh3 ${ }^{\mathrm{GD} /+}$ and Msh2-Msh3 ${ }^{\mathrm{GD} / \mathrm{GD}}$ nuclear extracts in lanes 8,9 and 10. Dissociation of DNA protein complexes was incomplete indicating that concentration of DNA and competitors needed to be adjusted.

\section{Abbreviations}

HNPCC: Hereditary non-polyposis colorectal cancer; LS: Lynch syndrome; Msh3: MutS homologue 3; MMR: mismatch repair; MSI: microsatellite instability.

\section{Acknowledgements}

Not applicable.

\section{Competing interests}

The authors declare that they have no competing interests.

\section{Availability of data and materials}

The datasets used during the current study are available from the corresponding author.

\section{Consent for publication}

Not applicable.

\section{Ethics approval}

The Institute of Animal Care and Use Committee (IACUC) of The Albert Einstein College of Medicine approved all animal experiment procedures. All the mice used were maintained in a dedicated animal care facility at the IACUC of the Albert Einstein College of Medicine according to IACUC rodent guidelines. All animal work was completed at the Albert Einstein College of Medicine.

\section{Funding}

This study was supported by The National Institute of Health grants CA76329 and CA93484 (WE).

\section{Publisher's Note}

Springer Nature remains neutral with regard to jurisdictional claims in published maps and institutional affiliations. 
Received: 19 August 2017 Accepted: 15 November 2017

Published online: 25 November 2017

\section{References}

1. Stojic L, Brun R, Jiricny J. Mismatch repair and DNA damage signalling. Bridge broken ends — cell. Response DNA Breaks Health Dis. 2004;3:1091-101.

2. Bellacosa A. Functional interactions and signaling properties of mammalian DNA mismatch repair proteins. Cell Death Differ. 2001:8:1076-92.

3. Martín-López JV, Fishel R. The mechanism of mismatch repair and the functional analysis of mismatch repair defects in Lynch syndrome. Fam Cancer. 2013;12:159-68

4. Li G-M. Mechanisms and functions of DNA mismatch repair. Cell Res. 2008:18:85-98.

5. Lamers MH, Winterwerp HH, Sixma TK. The alternating ATPase domains of MutS control DNA mismatch repair. EMBO J. 2003:22:746-56.

6. Lin DP, Wang Y, Scherer SJ, Clark AB, Yang K, Avdievich E, et al. An Msh2 point mutation uncouples DNA mismatch repair and apoptosis. Cancer Res. 2004;64:517-22.

7. Yang G, Scherer SJ, Shell SS, Yang K, Kim M, Lipkin M, et al. Dominant effects of an Msh6 missense mutation on DNA repair and cancer susceptibility. Cancer Cell. 2016;6:139-50.
8. Edelmann W, Umar A, Yang K, Heyer J, Kucherlapati M, Lia M, et al. The DNA mismatch repair genes Msh3 and Msh6 cooperate in intestinal tumor suppression. Cancer Res. 2000;60:803-7.

9. Glazer PM, Sarkar SN, Chisholm GE, Summers WC. DNA mismatch repair detected in human cell extracts. Mol Cell Biol. 1987:7:218-24.

10. Edelmann W, Cohen PE, Kane M, Lau K, Morrow B, Bennett S, et al. Meiotic pachytene arrest in MLH1-deficient mice. Cell. 1996:85:1125-34.

11. Larcombe-McDouall J, Buttell N, Harrison N, Wray S. In vivo pH and metabolite changes during a single contraction in rat uterine smooth muscle. J Physiol. 1999;518:783-90.

12. Gologan A, Sepulveda AR. Microsatellite instability and dna mismatch repair deficiency testing in hereditary and sporadic gastrointestinal cancers. Clin Lab Med. 2005;25:179-96.

13. Gupta S, Gellert M, Yang W. Mechanism of mismatch recognition revealed by human MutSbeta bound to unpaired DNA loops. Nat Struct Mol Biol. 2011:19:72-8.

14. Brown MW, Kim Y, Williams GM, Huck JD, Surtees JA, Finkelstein IJ. Dynamic DNA binding licenses a repair factor to bypass roadblocks in search of DNA lesions. Nat Commun. 2016;7:10607.

15. Sharma M, Predeus AV, Kovacs N, Feig M. Differential mismatch recognition specificities of eukaryotic MutS homologs MutSa and MutS $\beta$. Biophys J. 2014;106:2483-92.

16. Yamagishi H, Kuroda H, Imai Y, Hiraishi H. Molecular pathogenesis of sporadic colorectal cancers. Chin J Cancer. 2016:35:4

\section{Submit your next manuscript to BioMed Central and we will help you at every step:}

- We accept pre-submission inquiries

- Our selector tool helps you to find the most relevant journal

- We provide round the clock customer support

- Convenient online submission

- Thorough peer review

- Inclusion in PubMed and all major indexing services

- Maximum visibility for your research

Submit your manuscript at www.biomedcentral.com/submit

(OioMed Central 